\title{
CORRIGENDA IN VOLUME XXXV
}

Page 207 and elsewhere for "H. J. Wong" read "H. Y. Wong".

Page 219, line 12 from top-and last reference on page 220-for "Rao 1936" read "Rao 1938".

actual Dates of Publication for Volume XXXV

Numbers $1 / 2 \quad \ldots \quad$ 12th September, 1961

Numbers $3 / 4 \quad \ldots \quad$ 12th December, 1901

R. T. Leiper Supplement 17th April, 1961 